\title{
PROPUESTA DE ACCIONES PARA LA INTEGRACIÓN DE UN SISTEMA DE GESTIÓN AMBIENTAL CON EL SISTEMA DE GESTIÓN DE CALIDAD EXISTENTE EN UNA EMPRESA CONSULTORA
}

\author{
PROPOSED ACTIONS FOR THE INTEGRATION OF AN ENVIRONMENTAL \\ MANAGEMENT SYSTEM WITH EXISTING QUALITY MANAGEMENT SYSTEM \\ IN A CONSULTING FIRM
}

\author{
${ }^{1}$ Véronique Vera Maquet, ${ }^{2}$ Wilfredo Baldeón Quispe y ${ }^{3}$ Antonio Carpio Salinas
}

\begin{abstract}
Resumen
El presente trabajo propone las acciones para integrar un sistema de gestión ambiental (SGA) con un sistema de gestión de calidad (SGC) existente en la empresa Vera \& Moreno S.A., Consultores de Ingeniería. Se realizaron visitas a las instalaciones de la empresa a fin de evaluar el SGC con relación a la norma ISO 9001:2008 y la situación inicial en base a los requisitos de la norma ISO 14001:2004. También se identificaron y evaluaron los aspectos e impactos ambientales más significativos de la organización. A partir de ello, se definió el plan de integración, elaborándose la documentación del SIG, el Manual del SIG, la política de calidad y medio ambiente, los procedimientos, instructivos y formatos propios de la norma ISO 14001:2004. Finalmente, se llevó a cabo la fase inicial de la implementación del nuevo SGA, mediante la definición y ejecución de objetivos, metas y programas ambientales y la realización de charlas de sensibilización y capacitación al personal.
\end{abstract}

Palabras clave: ISO 9001:2008, ISO 14001:2004, sistema de gestión de la calidad, sistema de gestión ambiental, sistema integrado de gestión.

\begin{abstract}
This paper proposes actions to integrate an environmental management system (EMS) with the existing certified quality management system (QMS) in the company Vera \& Moreno S.A., Consultores de Ingeniería. The company's facilities were visited in order to assess the QMS relative to ISO 9001:2008 and its current environmental situation based on the requirements of ISO 14001:2004. The most significant environmental aspects and impacts of the organization were also identified and assessed. Based on these findings, the integration plan was created, developing the IMS documents, the IMS Manual, the quality and environmental policy, procedures, instructions and forms proper to ISO 14001:2004. Finally, the initial phase of the implementation of the new EMS was carried out, through the definition and execution of goals, targets and environmental programs, and conducting staff awareness and training sessions.
\end{abstract}

Key words: ISO 9001:2008, ISO 14001:2004, quality management system, environmental management system, integrated management system.

\section{Introducción}

Si el Sistema de Gestión de Calidad (SGC) basado en la norma ISO 9001:2008, busca mejorar la competitividad de una compañía mediante la satisfacción de las necesidades de sus clientes y la mejora continua de sus productos o servicios (ISO 9001:2008; López, S. 2006), el Sistema de Gestión Ambiental (SGA) basado en la norma ISO 14001:2004, permite mejorar el rendimiento ambiental de la empresa previniendo la contaminación y el cumplimiento de la legislación ambiental vigente aplicable a la organización (Block y Marash. 2002; Granero y Sánchez, 2007; Zaro, 2002; Rodríguez, 2011). Hoy en día, cada vez más empresas tienden a implementar el SGA con la finalidad básica de tener bajo control los efectos ambientales de sus actividades, productos o servicios (Seoánez y Angulo, 1999).
La integración de ambos sistemas de gestión es posible debido a la compatibilidad que existe entre ambas normas, las cuales exigen varios requisitos comunes, evitando la duplicidad y reduciendo la complejidad. La integración permite racionalizar el uso de los recursos, trabajar con un solo sistema de documentos, mantener políticas y objetivos organizacionales integrados, y mejorar la imagen de la compañía (INDICOPI, 2006. NTP 833.906 2006; Carmona, Buiza, Vázquez y Rivas, 2008).

Es por ello que el objetivo del presente trabajo es proponer un modelo de integración que detalle las actividades a desarrollar para implementar e integrar el sistema de gestión ambiental al sistema de gestión de la calidad existente en la empresa Vera \& Moreno S.A. Para ello se comenzó con una evaluación de la situación inicial del SGC y un diagnóstico base de su desempeño ambiental, 
seguido del desarrollo de la base documentaria y el manual del sistema integrado de gestión (SIG), para lo cual se adaptaron los documentos comunes a las normas ISO 9001:2008 e ISO 14001:2004 y se elaboraron documentos propios del SGA para el SIG de la empresa consultora, para finalmente definir las actividades a llevarse a cabo para lograr la implementación del mismo.

\section{Materiales y métodos}

El estudio se realizó en las instalaciones de la empresa Vera \& Moreno S.A, Consultores de Ingeniería, ubicada en la Av. José Leal $N^{\circ} 1526$, distrito de Lince, provincia y departamento de Lima. La metodología desarrollada se basa en 4 etapas, ver Fig.1.

\begin{tabular}{|c|c|c|c|}
\hline $\begin{array}{c}\text { ETAPA } 1 \\
\text { Diagnóstico Ambiental Inicial }\end{array}$ & $\begin{array}{c}\text { ETAPA } 2 \\
\text { Plan de Integración }\end{array}$ & & \\
\hline $\begin{array}{l}\text { Reunión inicial con } \\
\text { Gerencia y RED } \\
\text { Visita de campo a } \\
\text { instalaciones } \\
\text { - Revisión de } \\
\text { documentación SGC } \\
\text { - Recorrido por la empresa } \\
\text { - Entrevistas con el } \\
\text { personal } \\
\downarrow \\
\text { Aplicación de lista } \\
\text { de verificación ISO } \\
\text { 14001:2004 } \\
\downarrow \\
\text { Identificación de aspectos } \\
\text { y evaluación de impactos } \\
\text { ambientales } \\
\downarrow \\
\downarrow \\
\text { Elaboración del Diagnóstico } \\
\text { Ambiental Inicial }\end{array}$ & $\begin{array}{c}\text { Apoyo de alta dirección } \\
\text { Cronograma de actividades } \\
\text { para implantación del plan } \\
\text { de integración } \\
\uparrow \\
\text { Definición de nueva } \\
\text { organización del SIG } \\
\text { Grado de cumplimiento de } \\
\text { ISO 14001:2004 } \\
\text { † } \\
\text { Organización del SGC } \\
\text { † } \\
\text { Belección del tipo de } \\
\text { integración } \\
\text { Beneficios y dificultades de } \\
\text { la integración }\end{array}$ & $\begin{array}{c}\text { Elaboración del Manual } \\
\text { Integrado de Gestión } \\
\downarrow \\
\text { Adaptación de } \\
\text { documentos del SGC } \\
\text { al SIG } \\
\downarrow \\
\text { Elaboración de } \\
\text { documentos propios del } \\
\text { SGA } \\
\downarrow \\
\text { Definición de objetivos, } \\
\text { metas y programas } \\
\text { ambientales } \\
\downarrow \\
\text { Inicio de } \\
\text { implementación de } \\
\text { objetivos, metas y } \\
\text { programas ambientales }\end{array}$ & 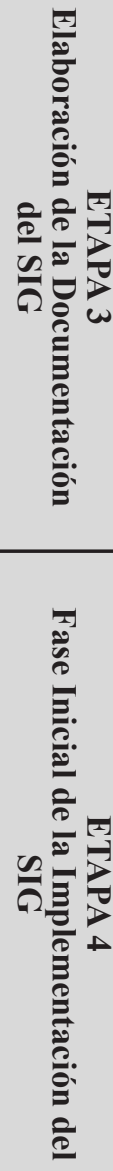 \\
\hline
\end{tabular}

Figura 1. Metodología para la integración de un SGA con un SGC existente.

ETAPA 1 - Evaluación de la situación inicial del SGC Y SGA

Al inicio del trabajo se tuvo una reunión con la Gerencia y el Representante de la Dirección (RED) para explicarles el objetivo del proyecto. Luego se realizaron visitas a las áreas e instalaciones de la empresa para identificar los procesos, actividades, prácticas ambientales y otros de la organización. Posteriormente se aplicó una lista de verificación para verificar el cumplimiento de los requisitos de la norma ISO 9001:2008. Dicha lista fue establecida por Pola y Palom (1997), citada por Gutiérrez y Meza (2013). Los resultados de la lista de verificación, se calificaron mediante la escala que se muestra en la Tabla 1.
Tabla 1. Escala de evaluación de la norma ISO 9001:2008.

\begin{tabular}{lcc}
\hline Puntuación & Observación & Significado \\
\hline 0 & No existe & $\begin{array}{c}\text { No se encontró nada. } \\
\text { Enfoque evidente en algunas } \\
\text { partes de la organización. }\end{array}$ \\
0.50 & $\begin{array}{c}\text { Existe algo } \\
\text { Existe en } \\
\text { grado mínimo } \\
\text { aceptable } \\
\text { Existe en } \\
\text { grado bueno }\end{array}$ & $\begin{array}{c}\text { Existen pautas definidas pero } \\
\text { no documentadas. } \\
\text { Documentado (manuales, } \\
\text { procedimientos, } \\
\text { reglamentos). }\end{array}$ \\
1 & $\begin{array}{c}\text { Existe } \\
\text { en grado } \\
\text { excelente }\end{array}$ & $\begin{array}{c}\text { Implantado, responde } \\
\text { completamente todos los } \\
\text { requisitos del sistema de } \\
\text { gestión de calidad. }\end{array}$ \\
\hline
\end{tabular}


Para la calificación final de cada requisito se aplicó la fórmula propuesta por Gutiérrez y Meza (2013), donde el numerador representa el puntaje alcanzado para un apartado, y el denominador, el puntaje máximo de dicho apartado.

$$
\text { Valorización }(\%)=\frac{\sum \text { Resultados obtenidos por apartado }}{2 \times \text { Número de preguntas del apartado }} \times 100
$$

Una vez realizadas las valorizaciones, se interpretaron los datos obtenidos de acuerdo a la siguiente clasificación (Gutiérrez y Meza, 2013):

\begin{tabular}{|c|c|c|c|}
\hline Deficiente & Regular & Bueno & Muy Bueno \\
{$[0 \%-50 \%] ;$} & {$[50 \%-70 \%] ;$} & {$[70 \%-90 \%] ;$} & {$[90 \%-100 \%]$} \\
\hline
\end{tabular}

Para conocer el grado de implementación inicial de la gestión ambiental de la empresa, se aplicó una lista de verificación en base a la norma ISO 14001:2004. El check list se basó en el documento "Herramientas para la gestión ambientalmente racional" del Bureau of International Recycling (Bureau of International Recycling - BIR, 2006). Para analizar el cumplimiento de los requisitos de la norma mencionada, se valoró la situación de la empresa utilizando la siguiente escala de puntuación: Cumplimiento nulo $=0$; Cumplimiento parcial $=1$; Cumplimiento completo $=2$.

$\mathrm{Si}$ bien el BIR propone sólo dos respuestas posibles a las preguntas de la lista de verificación: "SÍ" o "NO", en el presente trabajo se ha considerado por conveniente definir una escala numérica de puntuación para poder evaluar cuantitativamente los resultados. Además, se ha agregado en dicha escala un nivel de "cumplimiento parcial" con el fin de otorgar un valor a ciertos requisitos de la norma ISO 14001:2004 que están siendo cumplidos debido a que son comunes con la ISO 9001:2008, más no hacen referencia explícita al SGA, sino que son parte del SGC. Para calcular el porcentaje de cumplimiento, se adaptó la fórmula de la lista de verificación de la norma ISO 9001:2008, recordando que la valoración 2 representa el cumplimiento total de los requisitos:

La identificación de aspectos y evaluación de impactos ambientales (IAEIA) se realizó a partir de un registro de mapeo de procesos y utilizando la metodología desarrollada por la empresa REDOX Asesoría y Proyectos S.A.C., tomando como referencia los requisitos de la norma ISO 14001:2004 y las directrices de la norma ISO 14004:2004. En la Fig. 2 se muestra la matriz utilizada para el IAEIA.

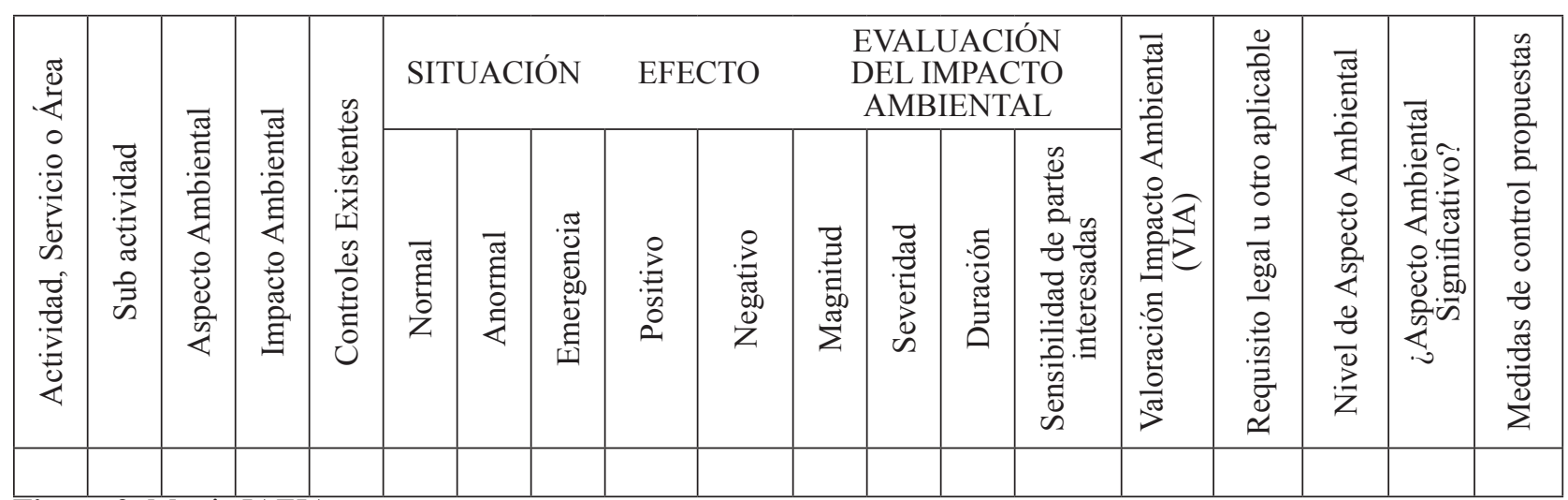

Figura 2. Matriz IAEIA.

En primer lugar, se identificaron los aspectos e impactos ambientales, observando las actividades que se llevan a cabo en las diferentes áreas de la empresa, así como los controles existentes. Luego, se evalúa cada uno de los impactos identificados, considerando los siguientes criterios:

- Criterios cualitativos de condición: condición normal, anormal o de emergencia.

- Criterios cualitativos de efectos:

Positivo: Produce un impacto beneficioso sobre el ambiente.
Negativo: Produce un impacto perjudicial sobre el ambiente.

- Criterios cuantitativos (Tabla 2): Magnitud (M), Severidad (S), Duración (D), Sensibilidad de Partes Interesadas (SPI). 
Tabla 2. Criterios para la determinación de factores.

\begin{tabular}{|c|c|c|c|}
\hline $\begin{array}{c}\text { Criterio de } \\
\text { significancia }\end{array}$ & Alto (3) & Medio (2) & Bajo (1) \\
\hline Magnitud (M) & $\begin{array}{l}\text { - La cantidad del residuo, } \\
\text { el uso del recurso o fuente } \\
\text { que genera el aspecto se } \\
\text { considera alto. } \\
\text { - Está por encima de los } \\
\text { estándares establecidos. }\end{array}$ & $\begin{array}{l}\text { - La cantidad del residuo, el uso } \\
\text { del recurso o fuente que genera el } \\
\text { aspecto se considera medio. } \\
\text { - Está por debajo de los estándares } \\
\text { establecidos. }\end{array}$ & $\begin{array}{l}\text { - La cantidad del residuo, el uso } \\
\text { del recurso o fuente que genera } \\
\text { el aspecto se considera mínimo } \\
\text { o no se produce. } \\
\text { - Está por debajo de los } \\
\text { estándares establecidos. }\end{array}$ \\
\hline Severidad (S) & $\begin{array}{l}\text { - Daños graves o irreversibles } \\
\text { al ambiente. } \\
\text { - Se manifiesta fuera de las } \\
\text { instalaciones de la empresa. } \\
\text { - Si ocurriese el aspecto } \\
\text { ambiental potencial se } \\
\text { considera el nivel de la } \\
\text { emergencia como Alto. }\end{array}$ & $\begin{array}{l}\text { - Afecta o afectaría } \\
\text { reversiblemente al ambiente. } \\
\text { - Afecta únicamente a las } \\
\text { instalaciones de la empresa. } \\
\text { - Si ocurriese el aspecto ambiental } \\
\text { potencial se considera el nivel de } \\
\text { la emergencia como Medio. }\end{array}$ & $\begin{array}{l}\text { - Hay una afectación mínima al } \\
\text { ambiente. } \\
\text { - Afecta solo a una parte de las } \\
\text { instalaciones de la empresa o } \\
\text { no afecta ninguna. } \\
\text { - Si ocurriese el aspecto } \\
\text { ambiental potencial se } \\
\text { considera el nivel de la } \\
\text { emergencia como Bajo. }\end{array}$ \\
\hline Duración (D) & $\begin{array}{l}\text { - La permanencia del impacto } \\
\text { ambiental es continua en } \\
\text { el ambiente o de larga } \\
\text { duración. } \\
\text { - El residuo o fuente que lo } \\
\text { genera no es degradable. } \\
\text { - La capacidad de } \\
\text { autodepuración es baja. }\end{array}$ & $\begin{array}{l}\text { - La permanencia del impacto } \\
\text { ambiental es interrumpida en el } \\
\text { ambiente (no es continua en el } \\
\text { tiempo). } \\
\text { - El residuo o fuente que lo genera } \\
\text { no es degradable. } \\
\text { - La capacidad de autodepuración } \\
\text { es lenta. }\end{array}$ & $\begin{array}{l}\text { - La permanencia del impacto } \\
\text { ambiental es mínima en el } \\
\text { ambiente o no es perceptible. } \\
\text { - El residuo o fuente que lo } \\
\text { genera es degradable. } \\
\text { - La capacidad de } \\
\text { autodepuración es alta. }\end{array}$ \\
\hline $\begin{array}{l}\text { Sensibilidad de las } \\
\text { partes interesadas - } \\
\text { (SPI) }\end{array}$ & $\begin{array}{l}\text { - Es percibido por las partes } \\
\text { interesadas como algo grave. } \\
\text { - Repercusión pública } \\
\text { alta, probables acciones } \\
\text { judiciales. }\end{array}$ & $\begin{array}{l}\text { - Las partes interesadas lo perciben } \\
\text { como medianamente grave. } \\
\text { - Repercusión pública media, } \\
\text { improbables acciones judiciales, } \\
\text { cartas de reclamos o papeletas. }\end{array}$ & $\begin{array}{l}\text { - Las partes interesadas no } \\
\text { se perciben afectadas por el } \\
\text { impacto real o potencial. } \\
\text { - Repercusión pública baja o } \\
\text { nula. }\end{array}$ \\
\hline
\end{tabular}

El índice de Valoración del Impacto Ambiental (VIA) se calculó a partir de:

$$
\mathbf{V I A}=\mathbf{M}+\mathbf{S}+\mathbf{D}+\mathbf{S P I}
$$

En base a dicho resultado, se definen las acciones a tomar respecto a los aspectos e impactos ambientales, como se presenta en la tabla 3.

Tabla 3. Valoración del Impacto Ambiental y Determinación de Acciones.

\begin{tabular}{|c|c|c|c|}
\hline \multicolumn{2}{|r|}{ No significativo } & \multicolumn{2}{|r|}{ Significativo } \\
\hline $\begin{array}{c}\text { Aceptable } \\
(\text { AC) } \\
(4-5)\end{array}$ & $\begin{array}{c}\text { No se requieren controles } \\
\text { adicionales. Se debe dar } \\
\text { consideración a soluciones más } \\
\text { efectivas a bajo costo o soluciones } \\
\text { que no aumenten más los costos. Se } \\
\text { requiere seguimiento para ver si se } \\
\text { mantienen los controles. }\end{array}$ & $\begin{array}{c}\text { Importante } \\
\text { (IM) } \\
(8-10)\end{array}$ & $\begin{array}{l}\text { Se deben establecer controles } \\
\text { estrictos e inmediatos sobre las } \\
\text { actividades que impliquen riesgo } \\
\text { ambiental. Si el riesgo ambiental } \\
\text { implica trabajos en marcha se } \\
\text { deben tomar acciones urgentes } \\
\text { comunicando al jefe inmediato. }\end{array}$ \\
\hline $\begin{array}{l}\text { Moderado } \\
\text { (MO) } \\
(6-7)\end{array}$ & $\begin{array}{c}\text { Se deben realizar esfuerzos para } \\
\text { reducir el riesgo ambiental, pero } \\
\text { los costos de prevención deben } \\
\text { ser medidos y limitados. Las } \\
\text { medidas de prevención deben } \\
\text { ser implementadas en periodos } \\
\text { definidos de tiempo. }\end{array}$ & $\begin{array}{c}\text { Pérdida Total } \\
\text { (PT) } \\
(11-12)\end{array}$ & $\begin{array}{l}\text { El trabajo no debe ser reanudado } \\
\text { hasta que el riesgo ambiental no } \\
\text { haya sido reducido. Si no es posible } \\
\text { reducir el riesgo ambiental, aún con } \\
\text { recursos ilimitados, el trabajo debe } \\
\text { permanecer prohibido. }\end{array}$ \\
\hline
\end{tabular}

Se califica como aspecto ambiental significativo a los aspectos que cumplen con uno o más de los siguientes requisitos: VIA $>7$ ó $\mathbf{S}=\mathbf{3}$.

Luego, se elaboró el diagnóstico ambiental inicial de la empresa conteniendo el resumen de la información levantada, el análisis de los resultados obtenidos de las etapas anteriores y las conclusiones respecto al desempeño ambiental base de la empresa (Roberts y Robinson, 1999). La estructura del documento fue la siguiente: i. Aspectos generales: Finalidad, Alcance, Metodología, Marco temporal, Referencias.

ii. Panorámica e información general: La compañía, El sitio de operaciones, Ubicación el sitio en relación con receptores de riesgos y alrededores.

iii. Revisión de las prácticas de gestión ambiental 
actuales.

iv. Revisión de actividades, productos y procesos desde un punto de vista ambiental: Descripción de los procesos principales del funcionamiento general del sitio de operaciones, Registro fotográfico, Inventario de equipos y bienes potencialmente asociados a aspectos ambientales, Descripción del consumo de recursos, Identificación de aspectos y evaluación de impactos ambientales.

v. Revisión de accidentes e incidentes ambientales previos

vi. Revisión de la legislación relevante

vii. Anexos

\section{ETAPA 2 - Desarrollo del plan de integración del SGA CON EL SGC}

Se elaboró el plan de integración, que incluyó la siguiente información [Block y Marash, 2002; AENOR, 2005):

Beneficios y dificultades de la integración.

Tipo de integración seleccionado (parcial o plena).

Organización del SGC con el que ya se trabajaba en la empresa.

- Resumen de cumplimiento de las normas ISO 14001:2004 e ISO 9001:2008.

- Nueva organización para el SIG a implantar.

- Cronograma de actividades para la implementación del plan.

Apoyo brindado por la alta dirección para la implementación del plan de integración.

\section{ETAPA 3 - Elaboración de la documentación del SIG}

En base al manual de calidad existente y los resultados del plan de integración, se elaboró el manual del SIG, que incluye los elementos clave que proporcionan una visión del conjunto del SIG. Junto con el manual, se presenta la política de calidad y medio ambiente.

Posteriormente, se modificaron los documentos comunes a ambas normas, identificados en el plan de integración, con el fin de incluir los requisitos de la norma ISO 14001:2004. Adicionalmente, se adaptaron los procedimientos y formatos propios del SGC para que hagan referencia al SIG, y no sólo a la gestión de la calidad.

En la modificación se los documentos se consideró las pautas indicadas en el procedimiento de control de documentos y registros de Vera \& Moreno S.A. en relación a la edición y la identificación de los cambios realizados en los documentos internos del sistema de gestión. Finalmente, se desarrollaron los documentos del SGA, definidos en el plan de integración.

\section{ETAPA 4 - Fase inicial de la implementación del SGA}

En base a los impactos ambientales identificados como significativos, se definieron objetivos y metas ambientales, con el fin de medir periódicamente su cumplimiento, permitiendo así valorar la eficacia del SGA y aplicar correcciones cuando fuese necesario. Asimismo, se definió el cronograma de capacitaciones para la formación del personal en temas ambientales y de calidad, y se establecieron los programas de sensibilización ambiental, con el fin de lograr la toma de conciencia del personal respecto a los aspectos ambientales significativos.

Con el fin de implementar los objetivos, metas y programas ambientales, se inició el cumplimiento de los programas de sensibilización ambiental, realizando todas las estrategias o actividades definidas; y se realizó la capacitación al personal sobre interpretación de la norma ISO 14001:2004 e identificación de aspectos y evaluación de impactos ambientales.

\section{Resultados y discusiones}

\section{Situación inicial del SGC}

Al aplicar de la lista de verificación del SGC, la empresa Vera \& Moreno S.A. obtuvo un puntaje global de $94.77 \%$, presentando un nivel de cumplimiento "muy bueno" de los requisitos de la norma ISO 9001:2008. En la Fig. 3 se observan los porcentajes obtenidos por capítulos de la norma.

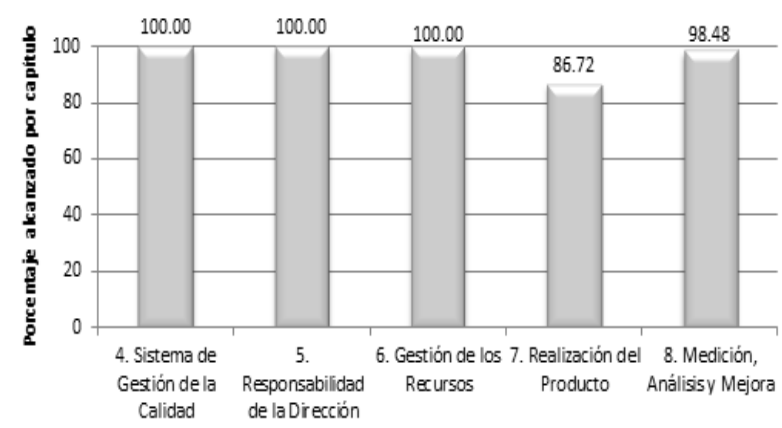

Figura 3. Porcentajes obtenidos de la lista de verificación ISO 9001:2008.

El SGC de la empresa ha sido implementado de manera adecuada, por lo que mantiene su certificación ISO 9001 desde el año 2006. Por ello, la mayoría de requisitos obtuvo una calificación "muy buena", con excepción del capítulo 7: Realización del Producto, cuya calificación fue "buena". En este último capítulo, se encontraron ciertas deficiencias, como la falta de una metodología documentada y la definición de responsables para la revisión de los requisitos relacionados con el producto (acápite 7.2), y la ausencia de registros del proceso de diseño y desarrollo (acápite 7.3). Además, en el capítulo 8: Medición, Análisis y Mejora, no se alcanzó el 100\% debido a que ciertas Solicitudes de Acción Correctiva o Preventiva (SAC) se han vuelto a generar, lo cual demuestra que no han sido realizados correctamente el análisis de causa y/o los planes de acción. 


\section{Diagnóstico ambiental inicial}

El resultado general de la aplicación de la lista de verificación del cumplimiento de la norma ISO 14001:2004 en la empresa Vera \& Moreno S.A. fue igual a $17.57 \%$ y los resultados de cada capítulo de la norma se presentan en la Fig. 4

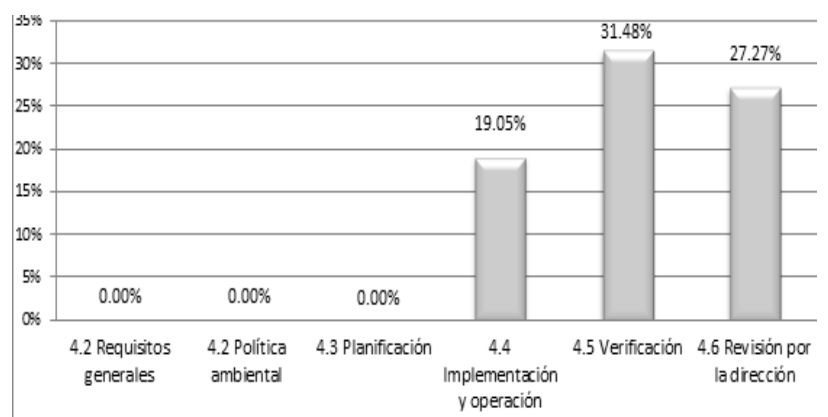

Figura 4. Porcentajes obtenidos de la lista de verificación ISO 14001:2004.

Dado que la empresa contaba con un SGC implementado, presentó ciertos avances respecto a algunos requisitos de la norma ISO 14001:2004 que son comunes a la norma ISO 9001:2008. Así, el capítulo más desarrollado fue el de verificación, donde se tenían los procesos de control de registros, auditoría interna y no conformidad, acción correctiva y acción preventiva. Asimismo, se llevaba a cabo el proceso de revisión por la dirección y algunos aspectos de la implementación y operación, como el control de documentos. Sin embargo, debido a que Vera \& Moreno S.A. no contaba con un SGA, todavía había mucho por hacer para lograr el cumplimiento de todos los requisitos de la norma ISO 14001:2004.

Por otro lado, la visita a las instalaciones permitió encontrar los siguientes malos hábitos ambientales en la empresa (ver tabla 4):

Tabla 4. Malos hábitos ambientales en Vera \& Moreno S.A.

\begin{tabular}{|c|c|}
\hline Manejo de residuos & $\begin{array}{c}\text { Consumo de energía } \\
\text { eléctrica }\end{array}$ \\
\hline $\begin{array}{l}\mathrm{A} \text { m a c e n a mi e n t o } \\
\text { inadecuado de materiales } \\
\text { puede malograrlos y } \\
\text { generar residuos. } \\
\text { ×Inadecuado acopio de } \\
\text { residuos y ausencia de } \\
\text { segregación. } \\
\times \text { Residuos de aparatos } \\
\text { eléctricos y electrónicos } \\
\text { sin disposición final } \\
\text { adecuada (computadoras, } \\
\text { impresoras, luminarias, } \\
\text { etc.). }\end{array}$ & $\begin{array}{l}\text { × Aparatos eléctricos } \\
\text { e n c h u f a d o s } \\
\text { constantemente. } \\
\text { × Cargador de celular } \\
\text { enchufado y sin utilizar. } \\
\text { × Luminarias encendidas } \\
\text { sin personal presente. } \\
\text { × Luminarias encendidas } \\
\text { durante el día, pudiendo } \\
\text { aprovechar la luz natural. } \\
\text { × Computadoras prendidas } \\
\text { cuando no se utilizan: } \\
\text { hora de almuerzo, salida } \\
\text { a reunión. etc. }\end{array}$ \\
\hline
\end{tabular}

Finalmente, se realizó la identificación de aspectos y evaluación de impactos ambientales, y se encontraron los siguientes aspectos ambientales significativos:

La generación de residuos sólidos peligrosos como tóners y cartuchos.

El consumo de energía eléctrica debido al uso de impresoras multifuncionales y computadoras.

El consumo de papel.

\section{PLAN DE INTEGRACIÓN}

El plan de integración fue elaborado con el fin de proponer acciones para integrar el SGA con el SGC con el que ya contaba la empresa.

En general, los principales beneficios de la integración son los siguientes:

Menores costos de mantenimiento: la gestión de ambos sistemas es más eficaz y eficiente debido a que comparten un gran número de elementos comunes.

Uso eficiente de los recursos: evita la duplicación de los recursos internos para gestionar el SGC y el SGA. Asimismo, reduce el tiempo empleado para a integración.

Reducción de la complejidad: mayor simplicidad en el diseño, implementación, mantenimiento y mejora de los sistemas. Asimismo, reduce la cantidad de documentación y mejora la implicación y la percepción de personal.

Mejora la comunicación interna, motivando al personal.

Mejora la imagen de la organización: el SIG se basa en las necesidades y expectativas de todas las partes interesadas, lo que demuestra su compromiso y mejora su imagen.

Mayor satisfacción de los clientes: gracias a la mejor calidad y desempeño ambiental en los productos y/o servicios.

Por el contrario, existen ciertas dificultades de integrar ambos sistemas de gestión:

Resistencia al cambio por parte del personal.

Necesidad de recursos adicionales (económicos y humanos) para planificar y ejecutar el plan de integración.

Mayor necesidad de formación del personal implicado en el SIG.

Se logró el nivel denominado "nivel integración" (Abad, 2009) e "integración total" [Block y Marash, 2002; Ministerio de Fomento, 2005), lo cual fue posible debido a que el alcance del SIG fue el mismo que el del SGC; la responsabilidad de ambos sistemas recayó sobre el mismo Representante de la Dirección (RED) del SGC; y el apoyo de la alta dirección permitió romper la resistencia al cambio en la organización.

La organización del SGC contaba con tres niveles de 
documentos: el primero describía el sistema, con la política de calidad, el manual de calidad y el perfil de puestos; el segundo agrupaba los procedimientos; y el tercero incluía los registros. El SGC no utilizaba ningún instructivo.

Con la integración del SGA con el SGC, se modificó el mapa de procesos para incluir los procesos del SGA, como la identificación de aspectos y evaluación de impactos ambientales y la atención de emergencias ambientales. Además, ya no se toma en cuenta solo a los clientes, sino a todas las partes interesadas, incluyendo al personal, los proveedores y la sociedad en general.

Asimismo, se re-estructuró la base documentaria del SIG, con cuatro niveles de documentos: el primero incluyó la política de calidad y medio ambiente, el manual del SIG y el perfil de puestos. En el segundo, se agregaron a los procedimientos existentes, 4 adicionales: Identificación de aspectos y evaluación de impactos ambientales; Identificación y evaluación del cumplimiento de requisitos legales y otros requisitos; Capacitación, formación y toma de conciencia; y Comunicación interna y externa. El tercer nivel agrupó dos nuevos instructivos: Preparación y respuesta ante emergencias ambientales, y Segregación de residuos. Finalmente, en el cuarto nivel se adjuntaron 5 registros adicionales: Matriz IAEIA, Matriz de requisitos legales, Cronograma de capacitaciones, Programa de sensibilización ambiental, Lista de verificación de inspecciones SOLE (Segregación, Orden y Limpieza, y Energía eléctrica).

El proyecto de integración contó con el apoyo de la alta dirección de Vera \& Moreno S.A., la cual se comprometió a aportar los recursos necesarios para llevar a cabo el plan de integración, ya sean recursos económicos, materiales o humanos, y a apoyar las acciones previstas, permitiendo la disponibilidad del personal para las entrevistas y actividades de capacitación posteriores.

\section{ELABORACIÓN DE LA DOCUMENTACIÓN DEL SIG}

Siguiendo lo establecido en el plan de integración, se elaboró el manual del SIG en base al manual de la calidad con el que ya contaba la empresa. Asimismo, se modificó la política de calidad para crear la política de calidad y medio ambiente, considerando los requisitos detallados en la norma ISO 14001:2004.

Además, se modificaron los documentos del SGC para incluir los requisitos de la norma ISO 14001:2004. Entre dichos documentos se encontraron cinco procedimientos (Acciones Correctivas, Acciones Preventivas y Producto No Conforme; Auditorías Internas; Control de Documentos y Registros; Compras; Gestión Comercial) y 12 registros. Finalmente, se elaboraron los documentos propios del SGA mencionados en el plan de integración.

\section{FASE INICIAL DE LA IMPLEMENTACIÓN DEL SGA}

Como primera etapa de la implementación del SGA, se adicionaron a los objetivos de calidad, los objetivos y metas ambientales presentados en la Tabla 5, los cuales fueron incluidos en el Tablero de Control, donde se define, para cada objetivo, el proceso al que hace referencia, el indicador para medirlo, las metas para cumplirlo, la frecuencia de medición, el plazo de cumplimiento y el responsable. En el caso de los objetivos ambientales, se define también el programa para lograrlos y su plazo de ejecución; y para los objetivos de calidad, los criterios de aceptación.

Tabla 5. Objetivos y metas ambientales.

\begin{tabular}{|c|c|c|c|}
\hline Objetivo & Indicador & Unidad de medida & Meta \\
\hline $\begin{array}{l}\text { Reducir el consumo de } \\
\text { recursos no renovables }\end{array}$ & $\begin{array}{l}\text { Consumo de energía eléctrica } \\
\text { por trabajador }\end{array}$ & $\mathrm{kWh} / \mathrm{hab}$ & $\begin{array}{l}\text { Reducir en un } 10 \% \text { con respecto al } \\
\text { semestre anterior }\end{array}$ \\
\hline Reducir el cc & Consumo de papel por ingreso & $\begin{array}{l}\text { Millar / } \\
\text { millón S/. }\end{array}$ & $\begin{array}{l}\text { Reducir en un } 10 \% \text { con respecto al } \\
\text { semestre anterior }\end{array}$ \\
\hline recursos & $\begin{array}{l}\text { Consumo de rollos para plotter } \\
\text { por ingreso }\end{array}$ & Número & $\begin{array}{l}\text { Reducir en un } 10 \% \text { con respecto al } \\
\text { semestre anterior }\end{array}$ \\
\hline Reducir la contaminación & Consumo de tóners por ingreso & Número / millón S/. & $\begin{array}{c}\text { Reducir en un } 10 \% \text { con respecto al } \\
\text { semestre anterior }\end{array}$ \\
\hline $\begin{array}{l}\text { del suelo por residuos } \\
\text { sólidos peligrosos }\end{array}$ & $\begin{array}{l}\text { Consumo cartuchos de tinta por } \\
\text { ingreso }\end{array}$ & Número / millón S/. & $\begin{array}{c}\text { Reducir en un } 15 \% \text { con respecto al } \\
\text { semestre anterior }\end{array}$ \\
\hline \multicolumn{2}{|c|}{$\begin{array}{l}\text { Desarrollar una cultura de HH de capacitaciones en cultura } \\
\text { reciclaje en la organización de reciclaje por mes }\end{array}$} & $\mathrm{HH}$ & $50 \mathrm{HH} / \mathrm{mes}$ \\
\hline
\end{tabular}

Asimismo, se establecieron dos programas de sensibilización ambiental: una para lograr la segregación de los residuos y el reciclaje de los mismos; y otro para concientizar al personal respecto al ahorro de energía eléctrica.

Luego, se ejecutaron todas las actividades de los programas de sensibilización ambiental:

Se instalaron recipientes de diferentes colores para papel, botellas de plástico PET y cartuchos de tinta y tóners.

Se elaboró el instructivo de segregación de residuos sólidos, que fue enviado vía correo electrónico al personal y publicado en los murales de las diversas áreas de la empresa.

Se realizó las charlas de sensibilización en materia de residuos sólidos y ahorro energético, a las cuales 
también participó la alta dirección.

Se realizaron las inspecciones SOLE en las diferentes áreas de la empresa y los resultados fueron enviados vía correo electrónico a todos los trabajadores, y publicados en los murales.

Se elaboró y difundió un decálogo de buenas prácticas de ahorro energético.

Además, se llevó a cabo la capacitación respecto a la interpretación de la norma ISO 14001:2004 e identificación de aspectos y evaluación de impactos ambientales, a cargo de un especialista externo. Se contactó también con la empresa HP para que recoja los tóners y cartuchos de tinta usados.

Todas las actividades mencionadas tuvieron como objetivo principal el buscar el cumplimiento de los objetivos y metas ambientales definidos en el Tablero de Control. Así, el personal se interesó por el contenido de las charlas y con el concurso en base a las inspecciones SOLE, aportando ideas, motivándose entre ellos mismos a cumplir con las buenas prácticas, lo cual se ve reflejado en los resultados de las inspecciones, al ver que éstos fueron más elevados en el mes de noviembre 2013, en comparación con el mes de octubre 2013.

\section{Conclusiones}

Se logró integrar el SGA al SGC que ya existía previamente en la empresa Vera \& Moreno S.A., por lo que el plan de integración se basó en una integración total o plena, contando con un solo manual del SIG y procedimientos comunes a ambos sistemas, lo que permitirá ahorrar recursos y tiempo.

La evaluación de la situación inicial del SGC alcanzó un resultado muy bueno de $94.77 \%$ de cumplimiento. A pesar de ello, el requisito 7.3 Diseño y Desarrollo obtuvo un puntaje regular debido a la ausencia de registros de dicho proceso.

Dado que la empresa Vera \& Moreno S.A no contaba con un SGA, la evaluación inicial del desempeño ambiental alcanzó un valor deficiente de $17.57 \%$ de cumplimiento, identificándose como aspectos ambientales significativos la generación de residuos sólidos peligrosos, el consumo de energía eléctrica y el consumo de papel.

Como producto del plan de integración, se elaboró el manual del SIG y la política de calidad y medio ambiente, modificándose además cinco de los ocho procedimientos existentes de calidad, así como doce registros, para incluir los requisitos de la norma ISO 14001:2004. Asimismo, se crearon cuatro procedimientos, dos instructivos y cinco registros adicionales específicos del sistema de gestión ambiental.

Siguiendo con la segunda etapa del proceso de integración, se implementaron los objetivos, metas y programas ambientales, realizando dos charlas de sensibilización ambiental y una capacitación sobre la norma ISO 14001:2004. Según lo definido en los programas, se distribuyó el material informativo de ahorro energético y segregación de residuos, para lo cual se instalaron cuatro recipientes para disponer los residuos sólidos de la empresa.

\section{Agradecimientos}

Nuestro profundo agradecimiento a la empresa Vera \& Moreno S.A., Consultores de Ingeniería, por todo el apoyo brindado en la elaboración y desarrollo del presente trabajo.

\section{Referencias}

Abad, J. 2009. Aspectos clave de la integración de sistemas de gestión. MC Salud Laboral. 4(4): 10-12.

AENOR (Asociación Española de Normalización y Certificación). 2005. UNE 66177:2005. Sistemas de gestión. Guía para la integración de los sistemas de gestión. Madrid. 25 p.

Block, M. y Marash, R. 2002. Integración de la ISO 14001 en un sistema de gestión de la calidad. 2da Edición. Ed. Fundación Confemetal, Madrid. 261 p.

Bureau of International Recycling - BIR. 2006. Herramientas para la gestión ambientalmente racional. Versión 8.0. Bruselas. 123 p. Consultado 22 de septiembre de 2013. Disponible en:

http://www.epa.gov/osw/conserve/materials/ecycling/ conference/resource/guide-esm-spanish.pdf

Carmona, M.; Buiza, G.; Vázquez, V. y Rivas, M. 2008. Guía para la integración de sistemas de gestión sobre la base de los procesos. Calidad, Medio Ambiente y Seguridad y Salud en el Trabajo. Instituto Andaluz de Tecnología, Sevilla. 208 p.

Granero, J. y Sánchez, M. 2007. Cómo implantar un Sistema de Gestión Ambiental según la norma ISO 14001:2004. 2da Edición. Ed. Fundación Confemetal, Madrid. 113 p.

Gutiérrez, S. y Meza, C. 2013. "Diseño de un Sistema de Gestión de Calidad para la Empresa Metal Mecánica GRUMETSAN S.A.C. a base de la Norma NTP-ISO 9001:2009". Trabajo de Investigación No Experimental de Ingeniero. Universidad Nacional Agraria La Molina. Lima - Perú. 74 p.

INDECOPI (Instituto Nacional de Defensa de la Competencia y de la Protección de la Propiedad Intelectual). 2006. NTP 833.9062006 . Guía de Aplicación de Sistemas Integrados de Gestión: ISO 9001, ISO 14001 y OHSAS 18001. Primera Edición. Perú. 60 p.

ISO. 2008. ISO 9001:2008. Sistema de Gestión de Calidad - Requisitos. ISO. 32 p.

López, S. 2006. Implantación de un sistema de calidad. Los diferentes sistemas de calidad existentes en la organización. 1 ${ }^{\text {era }}$ Edición. Ed. Ideaspropias, España. 176 p.

Ministerio de Fomento, España. 2005. Modelos para implementar la mejora continua en la gestión de 
empresas de transporte por carretera. Capítulo 3: Sistemas Integrados de Gestión. España. 13 p.

Roberts, H. y Robinson, G. 1999. ISO 14001 EMS:

Manual de Sistema de Gestión Medioambiental. 1era Edición. Ed. Paraninfo, Madrid. 425 p.

Rodríguez, A. 2011. Desarrollo sostenible y certificación - ISO 14001. Directorio Calidad Certificada en el Perú 2011. 3: $16-17$.

Seoánez, M. y Angulo, I. 1999. Manual de Gestión Medioambiental de la Empresa: Sistemas de Gestión Medioambiental, Auditorías Medioambientales, Evaluaciones de Impacto Ambiental y Otras Estrategias. Ed. Mundi Prensa Libros S.A., España. 515 p.

Zaro, L. 2002. ISO 14001. Guía para la implementación de la norma UNE-EN ISO 14001 en la pyme. Ed. Block C.I., Zaragoza. 92 p. 